\title{
Intérêt de la désinfection par photoactivation en endodontie
}

\section{RÉSUMÉ}

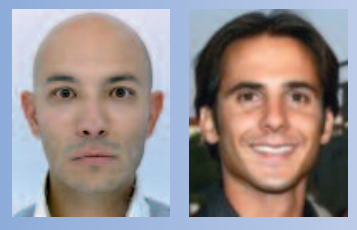

Raphaël DEVILLARD

Maître de conférences des Universités, praticien hospitalier,

UFR d'Odontologie,

Université Victor-Segalen Bordeaux 2,

16-20, cours de la Marne,

33082 Bordeaux cedex.

\section{Rodolphe ZUNZARREN}

Assistant hospitalier universitaire, UFR d'Odontologie,

Université Victor Segalen Bordeaux 2

La thérapeutique endodontique repose sur l'élimination des bactéries présentes dans le réseau canalaire. La complexité de la morphologie canalaire ne permet pas malgré les progrès réalisés par l'endodontie moderne, d'atteindre de façon systématique ce but. Dans le cas des lésions péri-apicales secondaires ou chroniques, la désinfection par photoactivation, qui utilise un agent photosensibilisant et une source laser de faible puissance, peut être un complément efficace à nos protocoles cliniques. Si son utilisation est facile et semble présenter un risque iatrogène limité, cette technique ne se substitue pas à une préparation chémomécanique de qualité mais représente une solution thérapeutique alternative.

- endodontie

- désinfection

- laser

- agents photosensibilisant / usage thérapeutique 
$>$

Il est accepté depuis longtemps que les pathologies péri-apicales d'origine endodontique sont principalement d'étiologies microbiennes [1]. Le principal objectif du traitement endodontique est donc d'obtenir l'élimination la plus complète possible de la charge bactérienne présente dans le réseau canalaire avant obturation.

Plusieurs études ont démontré qu'il existait une relation entre la quantité ainsi que le type de bactéries présentes dans l'endodonte au moment de l'obturation et le pronostic de guérison $[2,3]$.

Les principes modernes d'endodontie pour atteindre ce but se basent principalement sur le concept de préparation chemomécanique qui associe I'utilisation en rotation continue d'une instrumentation en alliage de nickel-titane et une irrigation à l'hypochlorite de sodium [4].

La complexité du réseau canalaire et l'organisation sous forme de biofilms des bactéries sont autant d'éléments limitant l'obtention de canaux exempts de présence bactérienne $[5,6]$. De fait, la préparation canalaire par rotation continue, indépendamment du choix de la séquence instrumentale, n'atteint pas l'ensemble des zones canalaires [7] et si l'irrigation est améliorée par l'association de systèmes ultrasonores d'activation améliorant la remontée des débris ainsi que le degré de pénétration de l'irrigant [8, 9], il semble extrêmement illusoire d'espérer obtenir une élimination complète des bactéries du réseau canalaire en une seule séance [10].

Des auteurs ont ainsi recommandé l'utilisation de médications interséances notamment de I'hydroxyde de calcium, afin d'atteindre un niveau de charge infectieuse le plus bas possible avant l'obturation [11, 12].

Le respect de l'ensemble du protocole opératoire incluant la mise en place d'un champ opé- ratoire ne garantit donc pas la certitude d'obtenir un canal stérile avant l'obturation.

Une solution complémentaire est d'utiliser la désinfection par photoactivation qui se base sur I'utilisation d'un laser de faible puissance et d'un agent photosensibilisant coloré.

L'utilisation de différents types de lasers a été proposée dans plusieurs domaines de la médecine buccodentaire : le diagnostic de lésions carieuses, la décontamination des poches parodontales ou le traitement de lésions muqueuses. Les lasers sont classés en fonction de la nature du milieu excité et de leur nocivité pour la vue qui est fonction de leur puissance et de leur longueur d'onde [13].

Dans le champ de l'endodontie, différentes longueurs d'ondes peuvent être utilisées avec des résultats encourageants [14] mais I'utilisation des lasers en tant que moyen complémentaire de désinfection reste controversée du fait de l'augmentation de température et de la vitrification qu'ils induisent [15] et également de leur intérêt par rapport à une irrigation à l'hypochlorite de sodium seule [16].

Une alternative élégante est la désinfection dite photosensible ou par photoactivation (DPA). Le principe de la DPA est d'utiliser un agent chimique photosensibilisant qui va sous l'action d'une source lumineuse de longueur d'onde définie produire des espèces réactives de l'oxygène tel que le radical superoxyde $0^{\circ}$ - et son acide conjugué, le radical hydroperoxyle $\mathrm{HO}_{2}{ }^{\circ}$, le radical hydroxyle $\mathrm{OH}^{\circ}$ et le peroxyde d'hydrogène $\mathrm{H}_{2} \mathrm{O}_{2}$ et induire la destruction de la cellule. Cette technique est déjà utilisée pour traiter différents carcinomes pulmonaires et œsophagiens en ciblant les cellules malignes sans avoir les effets indésirables des chimiothérapies [17].

L'application de cette technique au micro-organisme a été décrite il y a plus de 100 ans par 
Raab puis par von Tappeiner [18]. Supplantée par l'avènement des antibiotiques, la DPA redevient d'intérêt devant l'augmentation de cas de multirésistance aux antibiotiques et dans les cas où l'accès au site infectieux est complexe [19].

C'est donc naturellement qu'après avoir été proposée comme adjuvant thérapeutique dans le cadre des pathologies parodontales, la DPA a été appliquée au traitement endodontique [20]. Le protocole opératoire comprend l'application d'un agent photosensibilisant (AS) sous forme liquide à l'intérieur du réseau canalaire après la mise en forme. Cet agent, le plus souvent de la

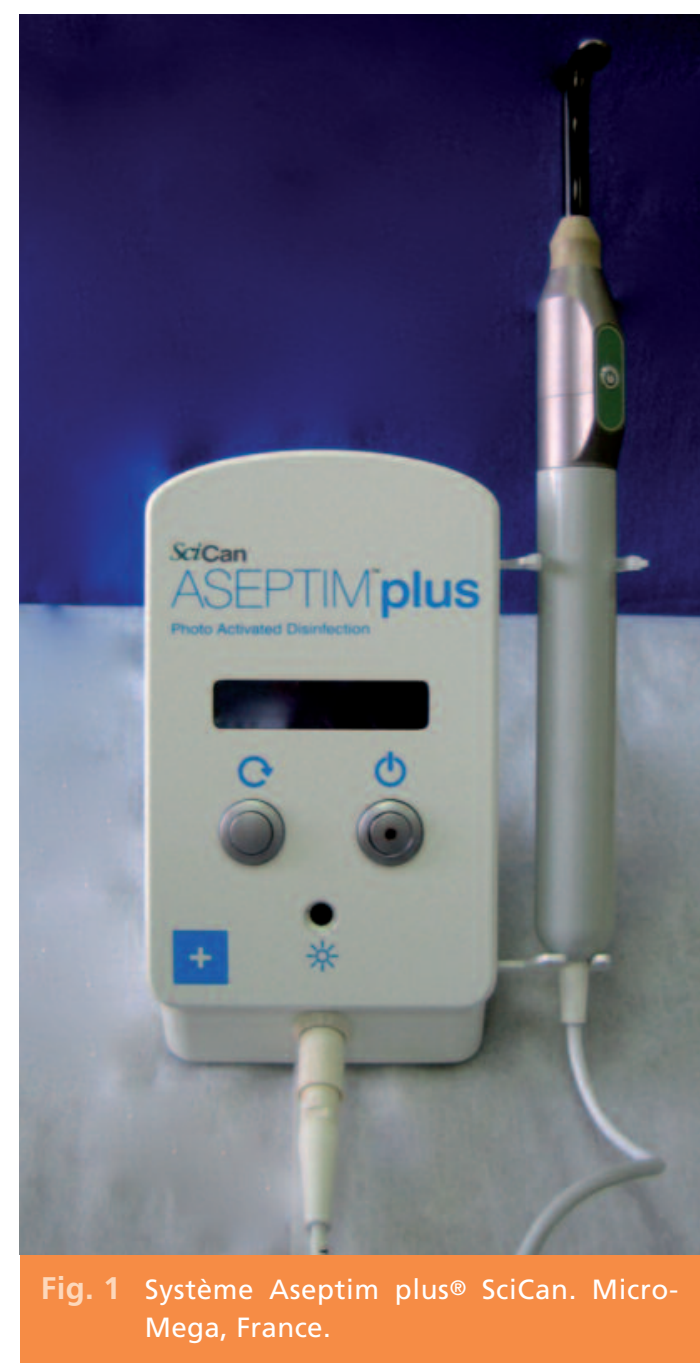

famille des phénothiazines, est du chlorure de tolonium ou du bleu de méthylène [18]. L'AS va se fixer aux bactéries Gram positives et négatives présentes avec différentes affinités, le chlorure de tolonium se fixant par exemple mieux à Enterococcus faecalis que le bleu de méthylène [20]. Deux modes d'action distincts vont alors conduire à la mort cellulaire par production d'espèces réactives de l'oxygène [21]. Le système comprend un générateur et une pièce à main (fig. 1) couplée à une fibre optique sur laquelle se fixeront des embouts spécifiques (fig. 2).

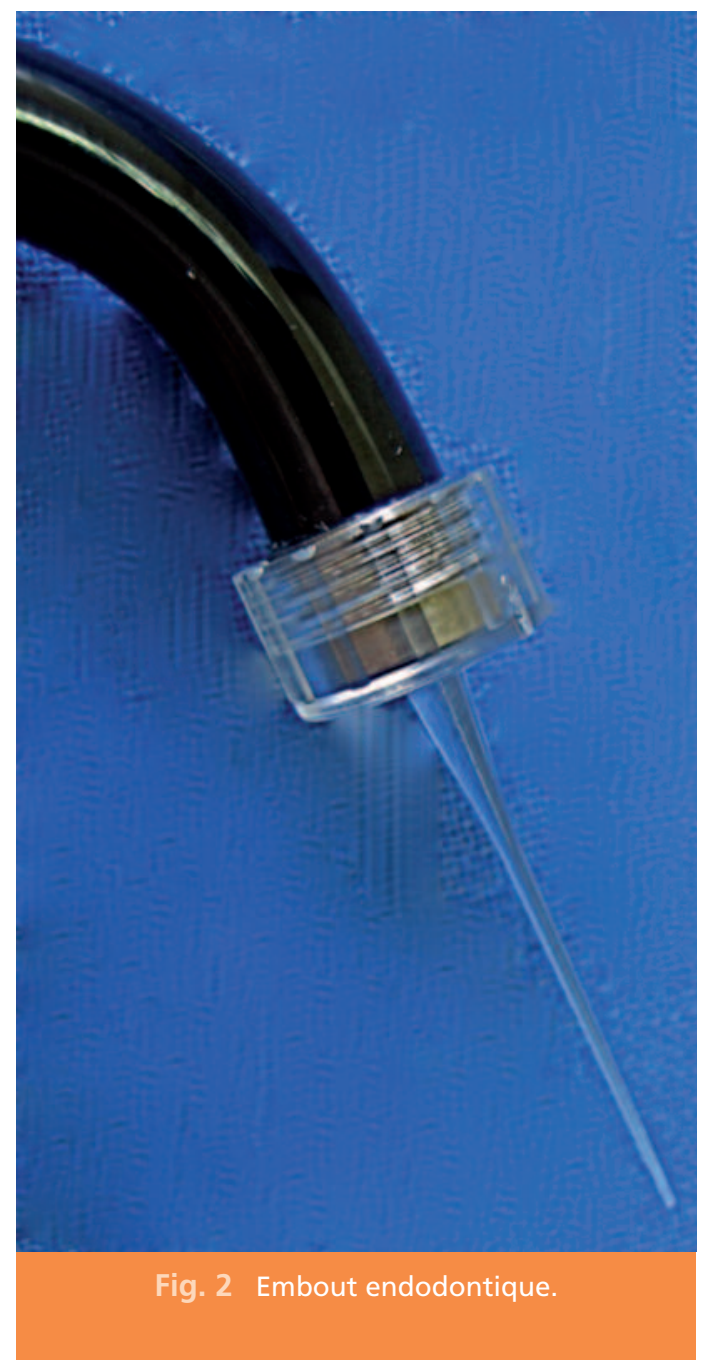




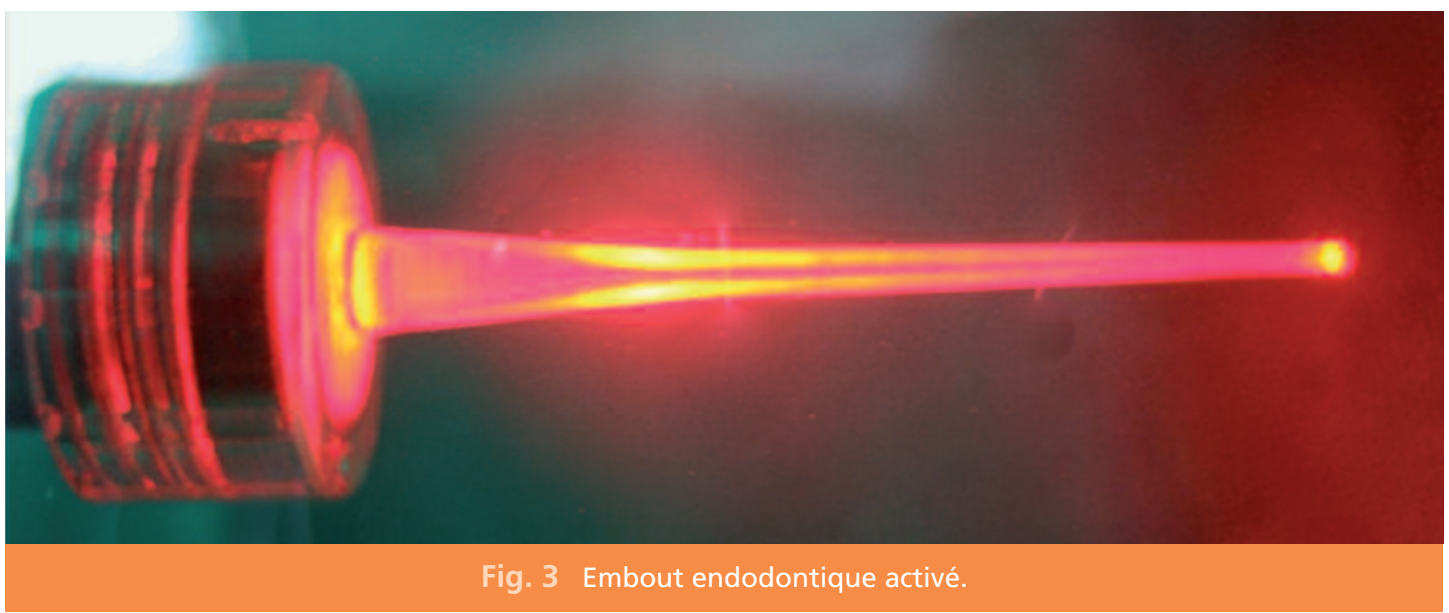

L'AS doit être placé dans l'ensemble du canal et laissé 60 secondes afin de permettre un contact optimal avec les bactéries. Du fait de ce temps de contact, l'agent ne doit pas présenter de cytotoxicité ou entraîner de coloration dentinaire permanente. II doit aussi être capable de pénétrer les tubuli dentinaires afin d'atteindre les bactéries.

Contrairement à l'hypochlorite de sodium qui présente une cytotoxicité non sélective, le bleu de toluidine ne semble pas avoir d'effet délétère sur les fibroblastes humains ou murins $[22$, 23] et même un effet positif sur des cellules odontoblastes-like [24].

Après élimination de l'enduit pariétal à l'aide d'EDTA ou d'acide citrique afin de libérer les tubuli et de permettre une pénétration optimale de I'AS, le canal est rincé à l'eau stérile puis séché à l'aide de pointe de papier. L'AS est ensuite mis en place dans les canaux et laissé en contact durant 60 secondes. On éliminera, lors de cette phase, les bulles d'air présentes dans le canal à l'aide d'une lime de diamètre fin afin de faciliter la propagation de la lumière. L'AS est ensuite activé dans chaque canal pendant 120 secondes à l'aide d'un embout conducteur flexible. Cette fibre va conduire une lumière rouge émise par le générateur de longueur d'onde de $635 \mathrm{~nm}$ et de puissance de $100 \mathrm{~mW}$ (fig. 3). À la différence des lasers de forte puissance, l'élévation de température est minime : $0,16{ }^{\circ} \mathrm{C}$ après $150 \mathrm{~s}$ d'exposition [25]. II est cependant nécessaire d'utiliser des moyens de protection oculaires pour le praticien et le patient (fig. 4).

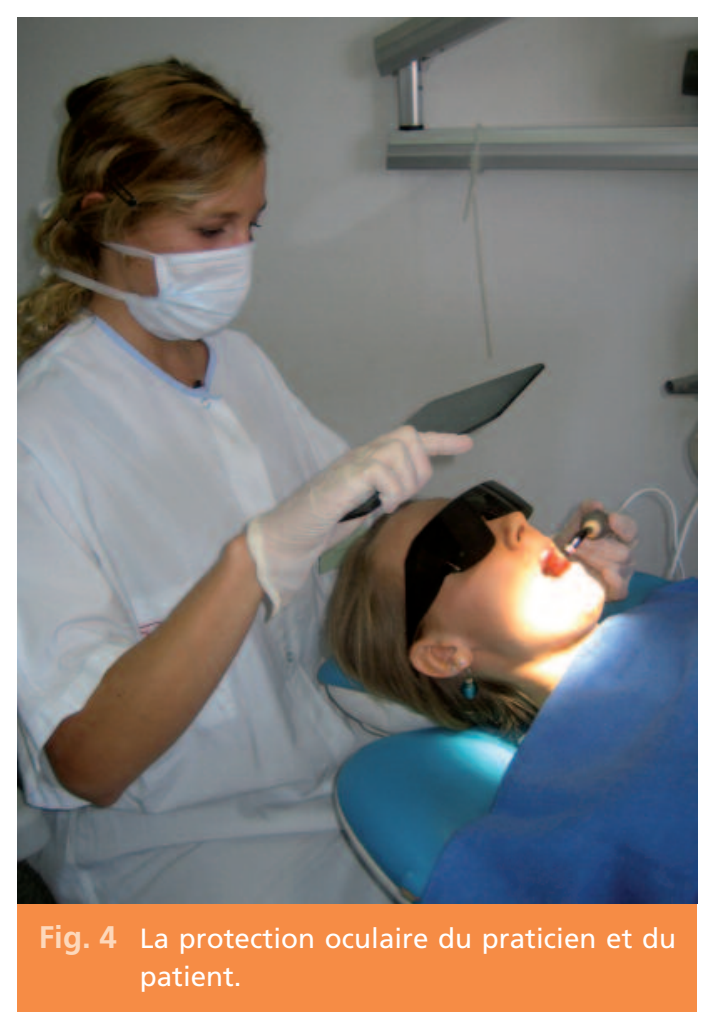


Le colorant est ensuite rincé grâce à de l'eau stérile et on réalisera une obturation endodontique classique.

Différentes études menées in vitro, ex vivo et in vivo ont mis en évidence l'efficacité de la DPA sur différentes bactéries sous forme planctonique ou organisées en biofilm [26-28]. Cependant pour l'équipe de Siqueira, la DPA nécessite encore des ajustements [29] et ne doit donc être envisagée que comme une thérapeutique adjuvante à la préparation chémomécanique [30].

La DPA représente donc une alternative prometteuse pour les cas cliniques complexes où elle sera utilisée en complément des thérapeutiques endodontiques traditionnelles. Les lésions secondaires et chroniques seront en particulier des cas où l'élimination complète des bactéries sera un prérequis à la cicatrisation.

Ces cas se retrouvant souvent lorsque les conditions anantomiques sont défavorables, la désinfection par photoactivation sera alors une alternative séduisante, la mise en forme du canal respectant les critères de diamètre et de conicité admis actuellement [31] permettra à l'irrigation mais également à l'agent photosensibilisant d'atteindre le tiers apical qui est la zone clé de la thérapeutique endodontique.

\section{Bibliographie}

1. Kakehashi S, Stanley HR, Fitzgerald RJ.

The effects of surgical exposures of dental pulps in germ-free and conventional laboratory rats. Oral Surg Oral Med Oral Pathol 1965 Sep;20:340-9.

2. Sakamoto $M$, Siqueira JF Jr., Rocas IN, Benno Y. Bacterial reduction and persistence after endodontic treatment procedures.

Oral Microbiol Immunol 2007 Feb;22(1):19-23.

3. Fabricius $L$, Dahlen $G$, Sundqvist G, Happonen RP, Moller AJ.

Influence of residual bacteria on periapical tissue healing after chemomechanical treatment and root filling of experimentally infected monkey teeth.
Eur J Oral Sci

2006 Aug;114(4):278-85.

4. Siqueira JF, Jr. Strategies to treat infected root canals.

$J$ Calif Dent Assoc 2001 Dec;29(12):825-37.

5. Ricucci $D$, Siqueira JF, Jr. Biofilms and apical periodontitis: study of prevalence and association with clinical and histopathologic findings. J Endod 2010 Aug;36(8):1277-88.

6. Nair PN, Henry S, Cano V, Vera J.

Microbial status of apical root canal system of human mandibular first molars with primary apical periodontitis after "one-visit» endodontic treatment. Oral Surg Oral Med Oral Pathol Oral Radiol Endod 2005 Feb;99(2):231-52.

7. Peters $\mathrm{OA}$, Schonenberger K, Laib A. Effects of four $\mathrm{Ni}-\mathrm{Ti}$ preparation techniques on root canal geometry assessed by micro computed tomography.

Int Endod J

2001 Apr;34(3):221-30.

8. de Gregorio $C$, Estevez R, Cisneros R, Paranjpe A, Cohenca N. Efficacy of different irrigation and activation systems on the penetration of sodium hypochlorite into simulated lateral canals and up to working length: an in vitro study.

$J$ Endod

2010 Jul;36(7):1216-21. 
9. Lee SJ, Wu MK, Wesselink PR.

The effectiveness of syringe irrigation and ultrasonics to remove debris from simulated irregularities within prepared root canal walls. Int Endod J 2004 Oct;37(10):672-8.

10. Sjogren U, Figdor D, Persson S, Sundqvist G. Influence of infection at the time of root filling on the outcome of endodontic treatment of teeth with apical periodontitis. Int Endod J 1997 Sep;30(5):297-306.

11. Shuping $G B$, Orstavik $D$, Sigurdsson A, Trope M. Reduction of intracanal bacteria using nickel-titanium rotary instrumentation and various medications. $J$ Endod 2000 Dec;26(12):751-5.

12. Kawashima N, Wadachi R, Suda $\mathrm{H}$, Yeng $\mathrm{T}$, Parashos $\mathrm{P}$. Root canal medicaments. Int Dent J 2009 Feb;59(1):5-11.

13. Walsh LJ.

The current status of laser applications in dentistry. Aust Dent J 2003 Sep; 48(3):146-55; quiz 98.

14. Schoop U, Kluger W, Dervisbegovic $S$, Goharkhay K, Wernisch J, Georgopoulos A, et al. Innovative wavelengths in endodontic treatment. Lasers Surg Med 2006 Jul;38(6):624-30.
15. Turkmen C, Gunday M, Karacorlu M, Basaran B. Effect of CO2, Nd:YAG, and ArF excimer lasers on dentin morphology and pulp chamber temperature: an in vitro study.

$J$ Endod

2000 Nov;26(11):644-8.

16. Le Goff A, Dautel-Morazin A, Guigand M, Vulcain JM, Bonnaure-Mallet $\mathrm{M}$.

An evaluation

of the $\mathrm{CO} 2$ laser

for endodontic disinfection.

$J$ Endod

1999 Feb;25(2):105-8.

17. Plaetzer $K$, Krammer $B$, Berlanda J, Berr F, Kiesslich T. Photophysics and photochemistry of photodynamic therapy: fundamental aspects.

Lasers Med Sci 2009 Mar;24(2):259-68.

18. Jori $G$, Fabris $C$, Soncin $M$, Ferro S, Coppellotti O, Dei D, et al.

Photodynamic therapy in the treatment of microbial infections: basic principles and perspective applications. Lasers Surg Med 2006 Jun;38(5):468-81.

19. Garcez AS, Nunez SC, Hamblim MR, Suzuki $\mathrm{H}$, Ribeiro MS.

Photodynamic therapy associated with conventional endodontic treatment in patients with antibiotic-resistant microflora: a preliminary report. $J$ Endod 2010 Sep;36(9):1463-6.
20. Lee MT, Bird PS, Walsh LJ. Photo-activated disinfection of the root canal: a new role for lasers in endodontics.

Aust Endod J 2004 Dec;30(3):93-8.

21. Ochsner M.

Photophysical

and photobiological processes in the photodynamic therapy of tumours. J Photochem Photobiol B 1997 May;39(1):1-18.

22. George $S$, Kishen $A$. Advanced noninvasive light-activated disinfection: assessment of cytotoxicity on fibroblast versus antimicrobial activity against Enterococcus faecalis.

$J$ Endod 2007 May;33(5):599-602.

23. $X u$ Y, Young MJ, Battaglino RA, Morse LR, Fontana $C R$,

Pagonis TC, et al. Endodontic antimicrobial photodynamic therapy: safety assessment in mammalian cell cultures. $\mathrm{J}$ Endod 2009 Nov;35(11):1567-72.

24. Oliveira CF, Basso FG, Lins EC, Kurachi $C$, Hebling J, Bagnato VS, et al. Increased viability of odontoblast-like cells subjected to low-level laser irradiation.

Lasers physics 2010;20(7):1659-66.

25. Dickers B, Lamard L, Peremans A, Geerts $S$, Lamy M, Limme M, et al. Temperature rise during photo-activated disinfection of root canals. 
Lasers Med Sci

2009 Jan;24(1):81-5.

26. Schlafer $S$, Vaeth $M$, Horsted-Bindslev $P$,

Frandsen EV.

Endodontic photoactivated

disinfection

using a conventional

light source: an in vitro

and ex vivo study.

Oral Surg Oral Med Oral Pathol Oral Radiol Endod 2010 Apr;109(4):634-41.

27. Bergmans $L$, Moisiadis $P$, Huybrechts B,

Van Meerbeek B, Quirynen M, Lambrechts P. Effect of photo-activated disinfection on endodontic pathogens ex vivo.
Int Endod J

2008 Mar;41(3):227-39.

28. Fimple JL, Fontana $C R$, Foschi F, Ruggiero K, Song $\mathrm{X}$, Pagonis TC, et al. Photodynamic treatment of endodontic polymicrobial infection in vitro.

$J$ Endod 2008 Jun;34(6):728-34.

29. Souza LC, Brito PR, de Oliveira JC, Alves FR, Moreira EJ, Sampaio-Filho HR, et al. Photodynamic therapy with two different photosensitizers as a supplement to instrumentation/irrigation procedures in promoting intracanal reduction of Enterococcus faecalis. J Endod 2010 Feb;36(2):292-6.

30. Lim Z, Cheng JL, Lim TW, Teo EG, Wong J, George S, et al. Light activated disinfection: an alternative endodontic disinfection strategy. Aust Dent J 2009 Jun;54(2):108-14.

31. Khademi A, Yazdizadeh M, Feizianfard M.

Determination

of the minimum instrumentation size for penetration of irrigants to the apical third of root canal systems. $J$ Endod 2006 May;32(5):417-20.

\section{SUMMARY}

\section{Photoactivated disinfection:}

\section{interest in endodontics}

Raphaël DEVILLARD

Rodolphe ZUNZARREN

\section{Keywords \\ - endodontic \\ - disinfection \\ - laser therapy \\ - photosensitizing \\ agents/therapeutic use}

The endodontic therapy is based on the elimination of bacteria in the root canal system. Despite the progress made in the endodontic field, the complexity of the root morphology does not allow to consistently achieve this goal. In the case of chronic or secondary periapical lesion, disinfection by photoactivation using a photosensitizing agent and a source of low power laser can be an effective complement to our clinical protocols. If its clinical use is easy and seems to present limited iatrogenic risk, this technic does not replace the fitting chemomecanical preparation but could be an alternative treatment option. 

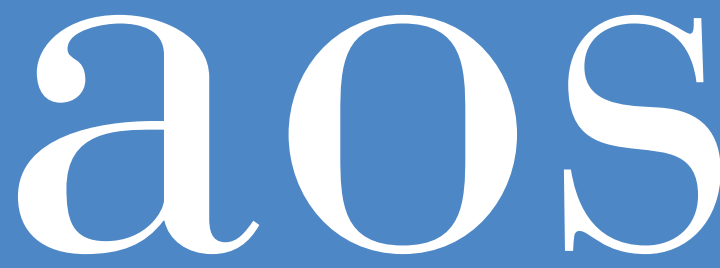

\section{Recommandations aux auteurs}

Les articles doivent être adressés en trois exemplaires au rédacteur-en-chef :

Dr Marc BERT, 1, rue de Chazelles - 75017 Paris.

Tout article doit être original et ne pas avoir déjà fait l'objet d'une publication.

Ils seront soumis au comité de rédaction des AOS qui, seul, acceptera, refusera ou demandera de modifier les textes qui lui sont proposés, en fonction de leur valeur scientifique ou clinique et de leur originalité.

Les auteurs préciseront leurs : nom, prénom, titres et affiliations universitaires ou hospitaliers éventuels, adresse, numéro de téléphone et de fax, ainsi que leur courriel.

\section{MANUSCRITS}

- dactylographier l'article en double interligne sur papier de format A4 (20 pages maximum) ;

- dactylographier les tableaux, légendes et références bibliographiques sur documents séparés ;

- le titre doit indiquer explicitement et avec le plus de concision possible le sujet du travail (60 signes, espaces compris) ; les intertitres ne doivent pas dépasser une demi-ligne ;

- joindre la traduction du titre de l'article en anglais ;

- joindre un résumé de 900 signes maximum (espaces compris) et sa traduction en anglais ;

- joindre 3 à 5 mots-clés choisis dans I'Index of Dental Litterature et sa traduction en anglais ;

- remettre trois exemplaires de la sortie laser ;

- joindre une copie en fichier informatique (texte enregistré de préférence sous Word 7).

\section{ICONOGRAPHIE}

- elle doit être originale et de bonne qualité. S'il s'agit d'une reproduction, une autorisation écrite de l'auteur de la photo, du schéma, de la radiographie, etc., doit être jointe avec les documents originaux;

- les schémas, dessins et radiographies doivent être réalisés à une échelle permettant une réduction ;

- chacune des illustrations doit comporter toutes les indications nécessaires (numéro de figure, nom du premier auteur, orientation). Les images en microscopie doivent comporter la mention du grossissement et l'échelle ;

- les figures doivent être numérotées et appelées par ce même numéro dans le texte ;

- les légendes des figures doivent être concises et figurer sur document séparé ;

- les documents iconographiques remis sous forme informatique sur CD-Rom devront être enregistrés sous Photoshop en JPG, EPS ou TIFF, résolution 300 dpi dans un format proche de celui souhaité dans la publication et être accompagnés de deux tirages papier en couleur.

\section{TABLEAUX}

- les présenter sur document séparé ;

- les légender ;

- les numéroter successivement en chiffres romains et les appeler dans le texte ;

\section{BIBLIOGRAPHIE}

Les références bibliographiques doivent être indexées dans le texte, présentées dans l'ordre de leur citation et nécessairement comporter les renseignements suivant l'ordre de la Convention dite de Vancouver :

\section{- pour un article de revue :}

Nom des auteurs, initiales des prénoms (Si plus de 6 auteurs, indiquer le premier suivi de et al.). Titre de la communication. Nom du journal (abrégé selon les normes internationales de I'Index Medicus, sans ponctuation après les abréviations) année;volume(numéro):pages (première et dernière pages).

Exemple : 1. Gary Bodo A, Merlier CH, Tarot P. Ostéosynthèse faciale par plaques miniaturisées à propos de 160 plaques du Pr Champy posées en un an. Ann Chir Plat Esthet 1983;28(1):41-47.

\section{- pour un article de livre :}

Même présentation des auteurs. Titre de l'article (dans la langue d'origine). In: noms et initiales des prénoms des " editors ». Titre de l'ouvrage. Lieu de publication : nom de l'éditeur, année:pages première et dernière pages.

Exemple : 2. Bokos JC. Deposition structure and properties of pyrolitic carbon. In: Walker PL, ed. Chemistry and physics of carbon. New York : Marcel Dekern, 1972:70-81.

\section{- pour un livre :}

Même présentation des auteurs. Titre de l'ouvrage. Lieu de publication : nom de l'éditeur, année.

Exemple : 3. Garber D, Goldstein R. Inlays et onlays en céramique et en composite ; restaurations postérieures esthétiques. Paris : Éditions CdP, 1995.

La terminologie employée devra permettre une compréhension indiscutée des termes choisis. Référence pourra être faite aux divers ouvrages professionnels existants.

Vingt-cinq tirés-à-part de chaque article seront adressés aux auteurs après publication. Toute demande de tirés à part supplémentaires devra être formulée au moment du bon à tirer.

En aucun cas, les AOS ne pourront être tenues responsables pour la perte, le vol ou la détérioration des textes et documents reçus. 